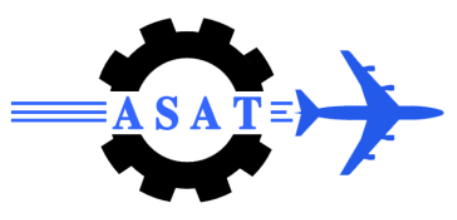

\title{
Quadrotor Autonomous Flight Control System Design
}

\author{
S. Robboz ${ }^{*}$, Y. Elhalwagy ${ }^{\dagger}$, O.E. Abdelhamid
}

\begin{abstract}
An autonomous quadrotor is an aerial helicopter with four horizontal rotors designed in a square configuration capable of locating lost or jeopardized victims, gathering military intelligence, and surveillance. Controlling of unmanned quadrotor is an interesting problems for control researchers. The literatures as assume a linear model and control it about operating point using the RPM, thrust, and torque In this paper, the proposed controllers are utilized on a full non linear model starting from voltages input able to determine its own attitude through an onboard sensor modeling to have a desired trajectory. The major goal of this ongoing research is the development and implementation of an autonomous flight control system for a quadrotor helicopter. Controlling a Vertical TakingOff and Landing (VTOL) flying vehicle is basically dealing with highly unstable dynamics and strong axes coupling. The simulation results showed a satisfactorily performance of the proposed controllers adopted on the quadrotor in face off the presence of coupling and nonlinearties. Vertical flight, hover, landing and horizontal flight are among the considered flight features in a predetermined trajectory.
\end{abstract}

Keywords: Quadrotor, Flight Control Systems

\section{Nomenclature:}

$\begin{array}{ll}\mathrm{g} & \text { gravitational acceleration. } \\ I & \text { Inertia matrix } \\ l & \text { the length between the center of blade } \\ & \text { and center of mass } \\ m & \text { total mass. } \\ \mathrm{q}, \mathrm{r} & \text { angler velocities in the body axis. }\end{array}$

$\mathrm{p}, \mathrm{q}, \mathrm{r}$ angler velocities in the body axis.

$\begin{array}{cl}x, y, z & \text { Coordinate of system } \\ V_{i} & \text { voltage of the motors } \\ v_{i} & \text { Torque of i rotor } \\ \phi, \theta, \psi & \text { Euler angles. } \\ \tau_{i} & \text { Thrust of i rotor }\end{array}$

\section{Introduction}

Last decades, due to their potential use on military and civil applications, unmanned aerial vehicles (UAV) became considerably popular. Today they are being used mainly for surveillance and inspection tasks. Nevertheless, recent advances in low-power embedded processors, miniature sensors and control theory are opening new horizons in terms of miniaturization and fields of use. Miniature Flying Robots (MFR) that use the Vertical Taking-Off and Landing concept (VTOL) have many advantages when compared to other mobile robots in complex or cluttered environments, [1] and commercial centers. MFRVTOL can also work on search-and-rescue missions after earthquakes, explosions, etc. An

\footnotetext{
* Syrian research student, Egypt.

${ }^{\dagger}$ Egyptian Armed Forces. Egypt.

* Professor of aerodynamics Egypt.
} 
aerial robot able to fly in narrow space and collapsed buildings can, for example, search victims of accidents or natural disasters without risking human lives[2].

The potential use of these flying robots and the challenges behind their development are attracting the scientific and the industrial community. Recently, many works in the literature outlined the MFR-VTOL mechanical design and the development of control strategies for maneuvers such as taking-off, hovering, and landing. Also, they are playing an important role in research areas like control engineering.

This paper presents an in progress development and implementation of an autonomous flight control system for a quadrotor helicopter. The developed closed loop control is for full nonlinear model with coupling challenges. Vertical flight, hover, landing and horizontal flight are among the simulated results. The paper organization is as follows; section 2 presents quadrotor dynamic. Section 3 discusses the quadrotors operation, section 4 is devoted to present the equation of motion and in the section 5 highlights the proposed control laws associated with the simulation results. The paper terminates with the conclusion.

\section{Quadrotor Dynamic Molding}

The quadrotor under consideration (Dragonfly XPro ) [3],[4],[5] consists of a rigid cross frame equipped with four rotors(see Figure 1). Pair of rotors 1-3 rotate in opposite direction to that pair 2-4, to cancel the gyroscopic torques [6],[7],[8], The up or down motion is achieved by increasing or decreasing the total thrust while maintaining an equal thrust of individual rotors. The forward/backward, left/right and the yaw motions are achieved through a differential control strategy of the thrust generated by each rotor[1],[2],[5],[6], [7].

The first step before the control development is an adequate dynamic system modeling [2], [8]. Especially for lightweight flying systems, the dynamic model ideally includes the gyroscopic effects resulting from both the rigid body rotation in space, and the four propeller's rotation.tip speed ratio and flapping angels and variables aerodynamics of thrust and torque. These aspects have been often neglected in previous works [2],[6].

Let us consider earth fixed frame $\mathrm{E}$ and body fixed frame B, using Euler angles parameterization, the airframe orientation in space is given by a rotation $\mathrm{R}$ from $\mathrm{B}$ to $\mathrm{E}$, where $\mathrm{R}$ is the rotation matrix [5],[6],[8],[9] as seen in figure(1). An experimental set up has been carried out for verification and validation of the 6-DOF model as well as the in progress research for controllers implementation as shown in Fig. (2).

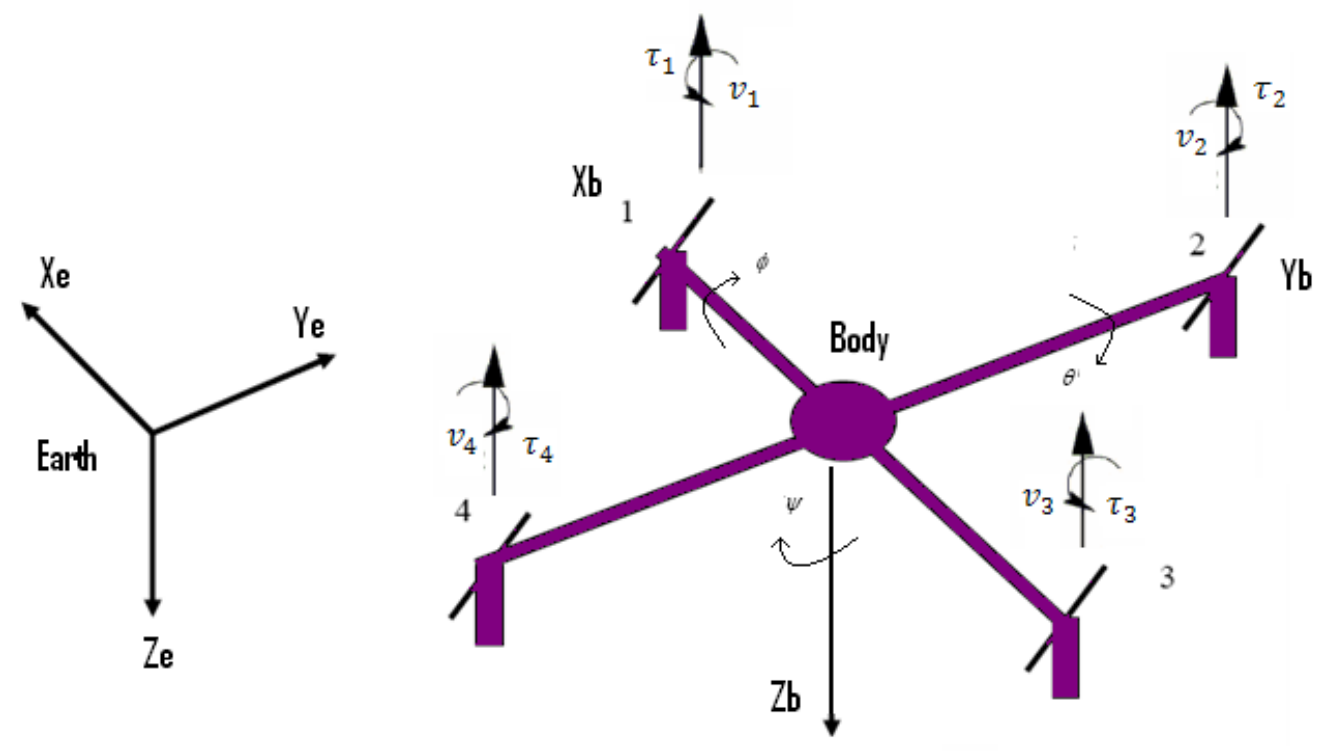

Figure (1) Alternative orientation for the body axes system and the same earth axes[7] 

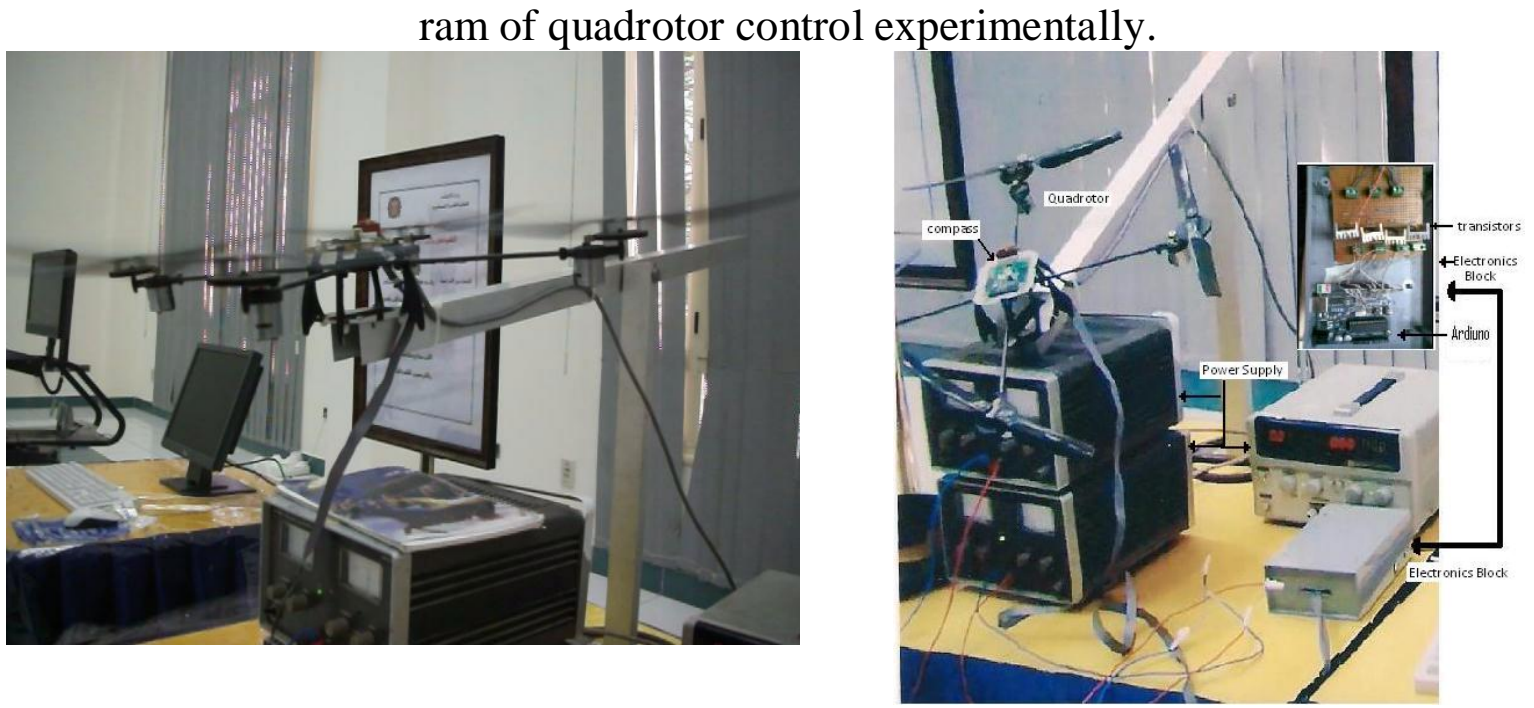

Figure (2) Experimental system flying

\section{Quadrotor Operation}

The quadrotor is controlled by independently varying the speed of the four rotors. Its mean that each rotor in a quadrotor is responsible for a certain amount of thrust and torque about its center of rotation, with these equations [3],[7]

The total thrust: $u_{1}=\tau_{1}+\tau_{2}+\tau_{3}+\tau_{4}, V_{1}+V_{2}+V_{3}+V_{4}$

The rolling moment: $u_{2}=l\left(\tau_{4}-\tau_{2}\right), \quad V_{4}-V_{2}$

The pitching moment: $u_{3}=l\left(\tau_{1}-\tau_{3}\right) \quad V_{1}-V_{3}$

The yawing moment: $u_{4}=v_{1}-v_{2}+v_{3}-v_{4}, V_{1}-V_{2}+V_{3}-V_{4}$

Quadrotor operation is shown in figure (4)[1],[10]

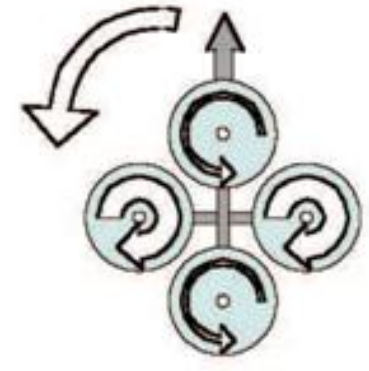

Rotate left

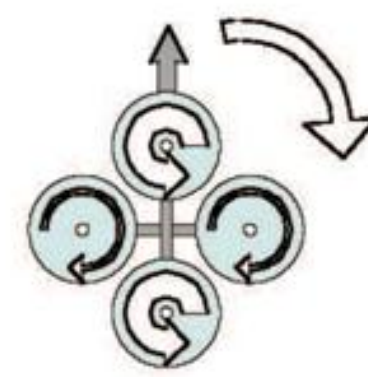

Rotate right

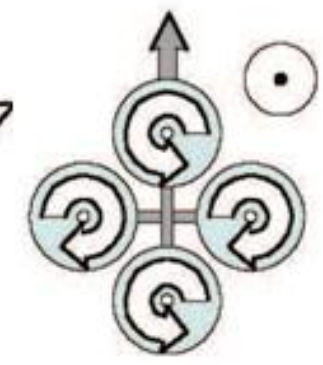

Going up

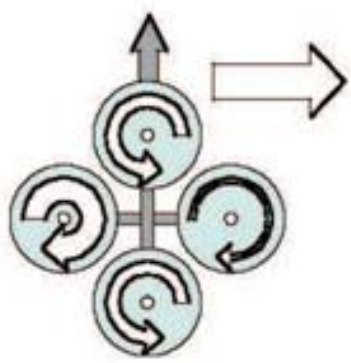

Move right

Figure (3) various movements of a quadrotor [1],[11]

\section{The Equations of Motion}

Using the Newton's law which is more comprehensible. The way of modeling the quadrotor differs from the one used for fixed wing vehicle in the fact that are not making the rotational transformations in the same order to go from the earth to body axes. Indeed, the most practical way is to carry out the final rotation of the earth to body transformation along the thrust direction [9]. Thus, we take for the body to earth transformation, the following direction cosine matrix: 
$R=\left[\begin{array}{ccc}S_{\theta} S_{\phi} S_{\psi}+C_{\psi} C_{\theta} & S_{\phi} S_{\theta} C_{\psi}-C_{\theta} S_{\psi} & S_{\theta} C_{\phi} \\ C_{\phi} S_{\psi} & C_{\psi} C_{\phi} & -S_{\phi} \\ C_{\theta} S_{\psi} S_{\phi}+S_{\theta} C_{\psi} & C_{\theta} S_{\phi} C_{\psi}+S_{\theta} S_{\psi} & C_{\theta} C_{\phi}\end{array}\right]$

where

$S_{\psi}=\sin (\psi), C_{\psi}=\cos (\psi), t_{\phi}=\tan (\phi)$

Thus, using $\left[\begin{array}{c}\ddot{x} \\ \ddot{y} \\ -\ddot{z}+g\end{array}\right]=R *\left[\begin{array}{c}0 \\ 0 \\ u_{1}\end{array}\right]$ we have

$$
\ddot{x}=-\frac{s_{\theta} C_{\phi}}{m} u_{1}, \ddot{y}=\frac{s_{\phi}}{m} u_{1} \quad \ddot{z}=-\frac{C_{\theta} C_{\phi}}{m} u_{1}+g
$$

where $\mathrm{x}, \mathrm{y}$ and $\mathrm{z}$ are the translational positions

Also, to relate Euler angular rates to body angular rates, we have to use the same order of rotation. This gives rise to:

$\left[\begin{array}{c}\dot{\phi} \\ \dot{\theta} \\ \dot{\psi}\end{array}\right]=\left[\begin{array}{ccc}C_{\psi} & -S_{\psi} & 0 \\ \frac{S_{\psi}}{c_{\phi}} & \frac{C_{\psi}}{C_{\phi}} & 0 \\ S_{\psi} t_{\phi} & C_{\psi} t_{\phi} & 1\end{array}\right]\left[\begin{array}{l}p \\ q \\ r\end{array}\right]$

Consider $I$ is the inertia matrix of the vehicle and $\vec{\omega}=\left[\begin{array}{l}p \\ q \\ r\end{array}\right]$

$\frac{d(I \vec{\omega})}{d t}=\left[\begin{array}{l}u_{2} \\ u_{3} \\ u_{4}\end{array}\right]=I\left[\begin{array}{l}\dot{p} \\ \dot{q} \\ \dot{r}\end{array}\right]+\vec{\omega} \wedge(I \vec{\omega}) \Rightarrow\left[\begin{array}{c}\dot{p} \\ \dot{q} \\ \dot{r}\end{array}\right]=I^{-1}(I \vec{\omega}) \wedge \vec{\omega}$

Assuming that the structure is symmetrical ([2] and [6]),

$$
I=\left[\begin{array}{ccc}
I_{x x} & 0 & 0 \\
0 & I_{y y} & 0 \\
0 & 0 & I_{z z}
\end{array}\right]
$$

In some papers, the second term of the right side of the above equation is neglected [8], [19]. This approximation can be made by assuming that:

The angular rate about the $\mathrm{z}$ axis, $\mathrm{r}$, is small enough to be neglected

$$
I_{x x}=I_{y y}
$$

Hence,

$$
\begin{aligned}
& \ddot{\phi}=-\dot{\psi} \dot{\theta} * c_{\phi}+\frac{c_{\psi}}{I_{x x}} u_{2}-\frac{s_{\psi}}{I_{y y}} u_{3}+\frac{I_{y y}-I_{z z}}{I_{x x}}\left(\dot{\psi}-\dot{\theta} * s_{\phi}\right) \dot{\theta} * c_{\phi} \\
& \ddot{\theta}=\frac{\dot{\psi} \dot{\theta}}{c_{\phi}}+\dot{\phi} \dot{\theta} * t_{\phi}+\frac{s_{\psi}}{c_{\phi} I_{x x}} u_{2}+\frac{c_{\psi}}{c_{\phi} I_{y y}} u_{3}-\frac{I_{y y}-I_{z z}}{I_{x x}}\left(\dot{\psi}-\dot{\theta} * s_{\phi}\right) \frac{\dot{\phi}}{c_{\phi}} \\
& \ddot{\psi}=\dot{\psi} \dot{\theta} * t_{\phi}+\frac{\dot{\phi} \dot{\theta}}{c_{\phi}}+\frac{s_{\psi} t_{\phi}}{I_{x x}} u_{2}-\frac{c_{\psi} t_{\phi}}{I_{y y}} u_{3}+\frac{1}{I_{z z}} u_{4}+\frac{I_{y y}-I_{z z}}{I_{x x}}\left(\dot{\psi}-\dot{\theta} * s_{\phi}\right) \dot{\phi} * t_{\phi}
\end{aligned}
$$




\section{Designing the Control Law}

\subsection{The State Space System}

The state vector is:

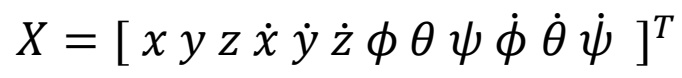

The input vector is:

$U=\left[\begin{array}{lll}u_{1} & u_{2 \_ \text {decoupled }} u_{2 \_ \text {decoupled }} u_{2 \_ \text {decoupled }}\end{array}\right]^{T}$

\subsection{Decupling Block}

After some experiments, it appears that the coupling between $\mathrm{x}, \mathrm{y}, \mathrm{z}$ and $\phi, \theta, u_{1}$ is not significant. Hence, The following set of equations about set point $u_{1-0}$ are considered [6]

$$
\begin{gathered}
\ddot{x}=-\frac{u_{1-0}}{m} \theta=-g \theta \\
\ddot{y}=\frac{u_{1-0}}{m} \phi=g \phi \\
\ddot{z}=\frac{1}{m}\left(u_{1}-u_{1-0}\right)
\end{gathered}
$$

Therefore, Only the coupling is considered between $\phi, \theta, \psi$ and $u_{2} u_{3} u_{4}$ as follow:

$$
\begin{gathered}
u_{2_{-} \text {decoupled }}=C_{\psi_{0}} u_{2}-\frac{I_{x x} s_{\psi_{0}}}{I_{y y}} u_{3}=I_{x x} \ddot{\phi} \\
u_{3 \_ \text {decoupled }}=\frac{I_{y y} s_{\psi_{0}}}{c_{\phi_{0}} I_{y y}} u_{2}+\frac{C_{\psi_{0}}}{c_{\phi_{0}}} u_{3}=I_{y y} \ddot{\theta} \\
u_{4_{-} \text {decoupled }}=\frac{I_{z z} s_{\psi_{0}} t_{\phi_{0}}}{c_{\phi_{0}} I_{x x}} u_{2}+\frac{I_{z z} c_{\psi_{0}} t_{\phi_{0}}}{I_{y y}} u_{3}-=I_{z z} \ddot{\psi}
\end{gathered}
$$

The transformation carried out in Simulink is:

$$
\left[\begin{array}{l}
u_{2} \\
u_{3} \\
u_{4}
\end{array}\right]=\left[\begin{array}{ccc}
C_{\psi_{0}} & S_{\psi_{0}} C_{\phi_{0}} \frac{I_{x x}}{I_{y y}} & 0 \\
-S_{\psi_{0}} \frac{I_{y y}}{I_{x x}} & C_{\psi_{0}} C_{\phi_{0}} & 0 \\
0 & -S_{\phi_{0}} \frac{I_{z z}}{I_{y y}} & 1
\end{array}\right]\left[\begin{array}{l}
u_{2_{-} \text {decoupled }} \\
u_{3 \_ \text {decoupled }} \\
u_{4_{-} \text {decoupled }}
\end{array}\right]
$$

\subsection{PID Controller}

The root locus method is used to design this controller. the gains of PID controller is shown in Table (1) ., Hence, the state space model is given as follows: 


$$
\dot{X}=\left[\begin{array}{cccccccccccc}
0 & 0 & 0 & 1 & 0 & 0 & 0 & 0 & 0 & 0 & 0 & 0 \\
0 & 0 & 0 & 0 & 1 & 0 & 0 & 0 & 0 & 0 & 0 & 0 \\
0 & 0 & 0 & 0 & 0 & 1 & 0 & 0 & 0 & 0 & 0 & 0 \\
0 & 0 & 0 & 0 & 0 & 0 & 0 & -g & 0 & 0 & 0 & 0 \\
0 & 0 & 0 & 0 & 0 & 0 & g & 0 & 0 & 0 & 0 & 0 \\
0 & 0 & 0 & 0 & 0 & 0 & 0 & 0 & 0 & 0 & 0 & 0 \\
0 & 0 & 0 & 0 & 0 & 0 & 0 & 0 & 0 & 1 & 0 & 0 \\
0 & 0 & 0 & 0 & 0 & 0 & 0 & 0 & 0 & 0 & 1 & 0 \\
0 & 0 & 0 & 0 & 0 & 0 & 0 & 0 & 0 & 0 & 0 & 1 \\
0 & 0 & 0 & 0 & 0 & 0 & 0 & 0 & 0 & 0 & 0 & 0 \\
0 & 0 & 0 & 0 & 0 & 0 & 0 & 0 & 0 & 0 & 0 & 0 \\
0 & 0 & 0 & 0 & 0 & 0 & 0 & 0 & 0 & 0 & 0 & 0
\end{array}\right] X+\left[\begin{array}{cccccc}
0 & 0 & 0 & 0 \\
0 & 0 & 0 & 0 \\
0 & & 0 & 0 & 0 \\
0 & & 0 & 0 & 0 \\
0 & & 0 & 0 & 0 \\
-1 / m & & 0 & 0 & 0 & \\
0 & 0 & 0 & 0 \\
0 & 0 & 0 & 0 \\
0 & 0 & 0 & 0 \\
0 & 1 / I_{x x} & 0 & 0 \\
0 & 0 & 1 / I_{y y} & 0 \\
0 & 0 & 0 & 1 / I_{z z}
\end{array}\right] U
$$

\subsection{LQR Controller}

Full-State Feedback and LQR pole placement control method are adapted in the design process. Linearized plant is given, to achieve a desired control, we must add poles and/or zeros to the system's forward path. By selecting the location of these poles carefully, we can achieve very tight tolerances to meet our specifications. This kind of controller aims to minimize the following quadratic cost function $J[1],[2],[10]$

$$
J=\int_{0}^{\infty}\left(X^{T}(t) Q X(t) U^{T}(t) R U(t)\right) d t
$$

where $\mathrm{Q}$ and $\mathrm{R}$ are weighting matrices

Using a feedback controller $\mathrm{K}$ such that $U(t)=-K X(t)$.. In Matlab relatively easy to work out the optimal controller $\mathrm{K}$ such that $\mathrm{J}$ is minimized. Indeed, if the state space representation of the system is $\dot{X}=A X+B U$ we have:

$$
K=R^{-1} B^{T} * P x
$$

where $\mathrm{P}$ is symmetric positive semidefinite solution of the Algebraic Riccati Equation(ARE) thanks to the Matlab command $\operatorname{are}(. .$.$) to solve this equation$

$$
0=P A+A^{T} P+Q-P B R^{-1} B P
$$

Consequently, the task in the LQR design is to choose appropriate weighting matrices.

$\mathrm{Q}$ and $\mathrm{R}$ are usually diagonal matrices. Coefficients of $\mathrm{Q}$ limit the amplitude of the state variables, coefficients of $\mathrm{R}$ limit the amplitude of the inputs.. The chosen weighting matrices are:

$$
Q=\left[\begin{array}{cccccccccccc}
10 & 0 & 0 & 0 & 0 & 0 & 0 & 0 & 0 & 0 & 0 & 0 \\
0 & 10 & 0 & 0 & 0 & 0 & 0 & 0 & 0 & 0 & 0 & 0 \\
0 & 0 & 10 & 0 & 0 & 0 & 0 & 0 & 0 & 0 & 0 & 0 \\
0 & 0 & 0 & 1 & 0 & 0 & 0 & 0 & 0 & 0 & 0 & 0 \\
0 & 0 & 0 & 0 & 1 & 0 & 1 & 0 & 0 & 0 & 0 & 0 \\
0 & 0 & 0 & 0 & 0 & 1 & 0 & 0 & 0 & 0 & 0 & 0 \\
0 & 0 & 0 & 0 & 0 & 0 & 1 & 0 & 0 & 0 & 0 & 0 \\
0 & 0 & 0 & 0 & 0 & 0 & 0 & 1 & 0 & 0 & 0 & 0 \\
0 & 0 & 0 & 0 & 0 & 0 & 0 & 0 & 10 & 0 & 0 & 0 \\
0 & 0 & 0 & 0 & 0 & 0 & 0 & 0 & 0 & 1 & 0 & 0 \\
0 & 0 & 0 & 0 & 0 & 0 & 0 & 0 & 0 & 0 & 1 & 0 \\
0 & 0 & 0 & 0 & 0 & 0 & 0 & 0 & 0 & 0 & 0 & 1
\end{array}\right], R=\left[\begin{array}{ccccc}
0.01 & 0 & 0 & 0 \\
0 & 0.1 & 0 & 0 \\
0 & 0 & 0.1 & 0 \\
0 & 0 & 0 & 0.1
\end{array}\right]
$$


The Q matrix has unitary coefficients except for the controlled variables ( $\mathrm{x}, \mathrm{y}, \mathrm{z}$ and $\psi$ ). Indeed, the priority is to make the UAV reach its desired position as fast as possible. In other terms, the main goal is to minimize the $L_{1}$ norm of $\mathrm{x}, \mathrm{y}, \mathrm{z}$ and $\psi$. Multiplying the associated coefficients by 10 highlights these variables and makes the cost function depend more significantly on them.

After different attempts, it appears that the dynamic performances are optimized with the above R matrix. This means that the input amplitudes don't really need to be minimized.

With these weighting matrices, the controller is in fact designed to minimize the $L_{2}-$ norm of $\mathrm{x}, \mathrm{y}, \mathrm{z}$ and $\psi$ without considering the other variables too much.

We build $m$ file to with this state in the Matlab to design of both PID and LQR controllers. The gains for PID and LQR controller is shown in table (1)

The model run for LQR simulations is the same, except that the feedback gains are different. is shown in figure (5)

Table (1) Gains for PID controller

\begin{tabular}{|c|c|c|c|c|c|c|c|c|}
\hline & \multicolumn{2}{|c|}{ Control of $x$} & \multicolumn{2}{|c|}{ Control of $y$} & \multicolumn{2}{|c|}{ Control of $z$} & \multicolumn{2}{|c|}{ Control of $\psi$} \\
\hline & PID & LQR & PID & LQR & PID & LQR & PID & LQR \\
\hline$\dot{\theta}$ & 5 & 4.3043 & & & & & & \\
\hline$\theta$ & 37.3 & 25,2884 & & & & & & \\
\hline$\dot{x}$ & -10.92 & $-7,8458$ & & & & & & \\
\hline$x$ & -10.9 & 10 & & & & & & \\
\hline$\dot{\phi}$ & & & 5 & 4.2976 & & & & \\
\hline$\phi$ & & & 37.3 & 25.2673 & & & & \\
\hline$\dot{y}$ & & & -10.92 & 7.8431 & & & & \\
\hline $\mathrm{y}$ & & & -10.9 & 10 & & & & \\
\hline$\dot{Z}$ & & & & & -10 & -15.7751 & & \\
\hline$Z$ & & & & & -10.6 & -31.6228 & & \\
\hline$\dot{\psi}$ & & & & & & & 3 & 3.9936 \\
\hline$\psi$ & & & & & & & 7.56 & 10 \\
\hline
\end{tabular}

\section{Simulation Results}

A complete non linear model for quadrotor flying vehicle has been carried out [4],[5]. Extensive simulations have been conducted for various flight scenarios. The reported results are for desired position inputs given for the case study where $x=3 m, y=-3 m, z=-4 m$.

\subsection{PID Controller}

Results show a satisfactorily performance for the utilized controller in the outer positioning loop (the desired displacements in $(\mathrm{x}, \mathrm{y}, \mathrm{z})$ ) as shown in figure $(5, \mathrm{~A}, \mathrm{~B}, \mathrm{C})$. It is clear that the linear velocity components $u_{e}, v_{e}$, and $w_{e}$ have non-zero value in the transient state as shown in figure $(5, \mathrm{D}, \mathrm{E}, \mathrm{F})$. The attitude behavior has shown the non-zero values in the transition from initial position to the desired position. The behavior of the roll $\phi$, pitch $\theta$ and yaw $\psi$ are presented in figure $(5, \mathrm{G}, \mathrm{H}, \mathrm{J}, \mathrm{K})$. However, the yaw angels $\psi$ are increased with small slope value but the velocity have constant value $\dot{\psi}=r=$ constant $\approx 10^{-5} \mathrm{rad} / \mathrm{sec}$ that may lead to dynamical coupling phenomena. 


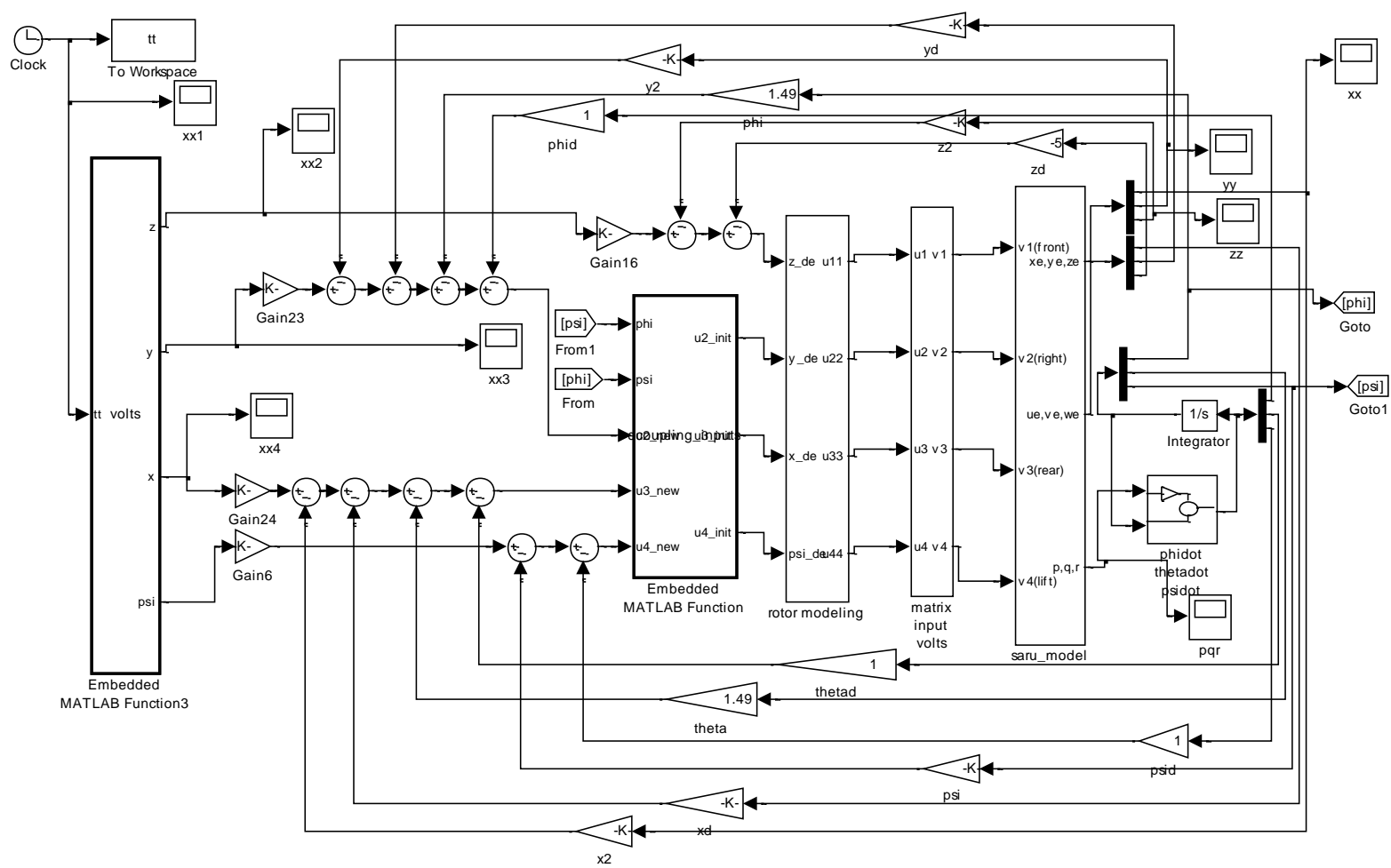

Figure(4) Simulation model PID LQR controller
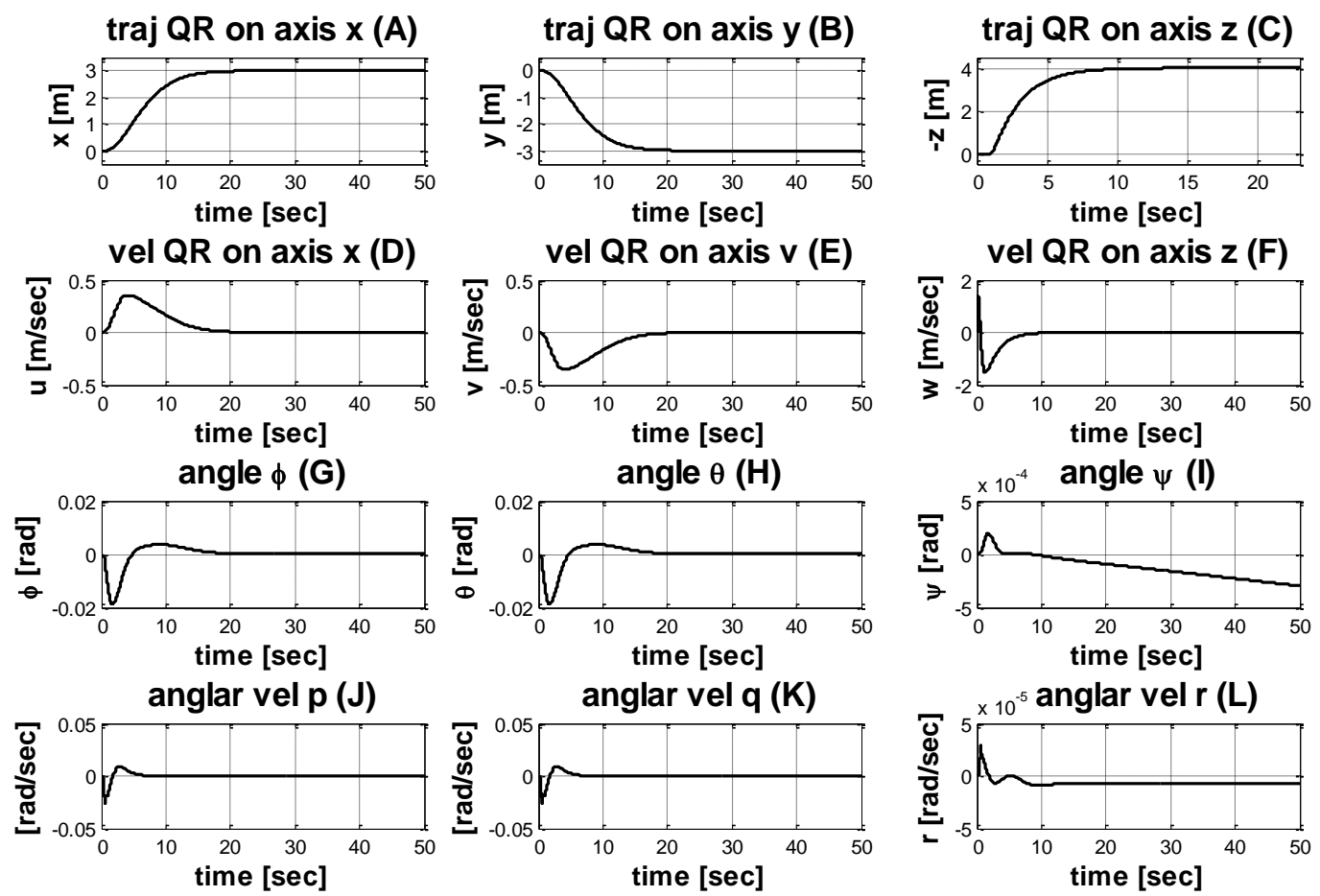

Figure (5) (A B C)linear positions(x, y, z), (D E F) linear velocities(u, v, w), ( G H I) angler positions $(\phi, \theta, \psi)$ and (J K L ) angler velocities ( p, q, r) 


\subsection{LQR controller}

Results show a satisfactorily performance for the utilized controller in the outer positioning loop (the desired displacements in $(\mathrm{x}, \mathrm{y}, \mathrm{z}))$ as shown in figure $(6, \mathrm{~A}, \mathrm{~B}, \mathrm{C})$. It is clear that the linear velocity components $u_{e}, v_{e}$, and $w_{e}$ have non-zero value in the transient state as shown in figure $(6, \mathrm{D}, \mathrm{E}, \mathrm{F})$. The attitude behavior has shown the non-zero values in the transition from initial position to the desired position. The behavior of the roll $\phi$, pitch $\theta$ and yaw $\psi$ are presented in figure $(6, \mathrm{G}, \mathrm{H}, \mathrm{J}, \mathrm{K})$. However, the yaw angels $\psi$ are increased with small slope value but the velocity have constant value $\dot{\psi}=r=$ constant $\approx 10^{-3} \mathrm{rad} / \mathrm{sec}$ that may lead to dynamical coupling phenomena. Its clear that the settling time is significantly reduced in comparable with the previously presented controller as shown in Figure (7). Yet, the simplicity of PID controller in hardware implementation may be considered. Optimal research engines e.g Genetic Algorithm (GA) is to be considered during the hardware implementation of the controller.
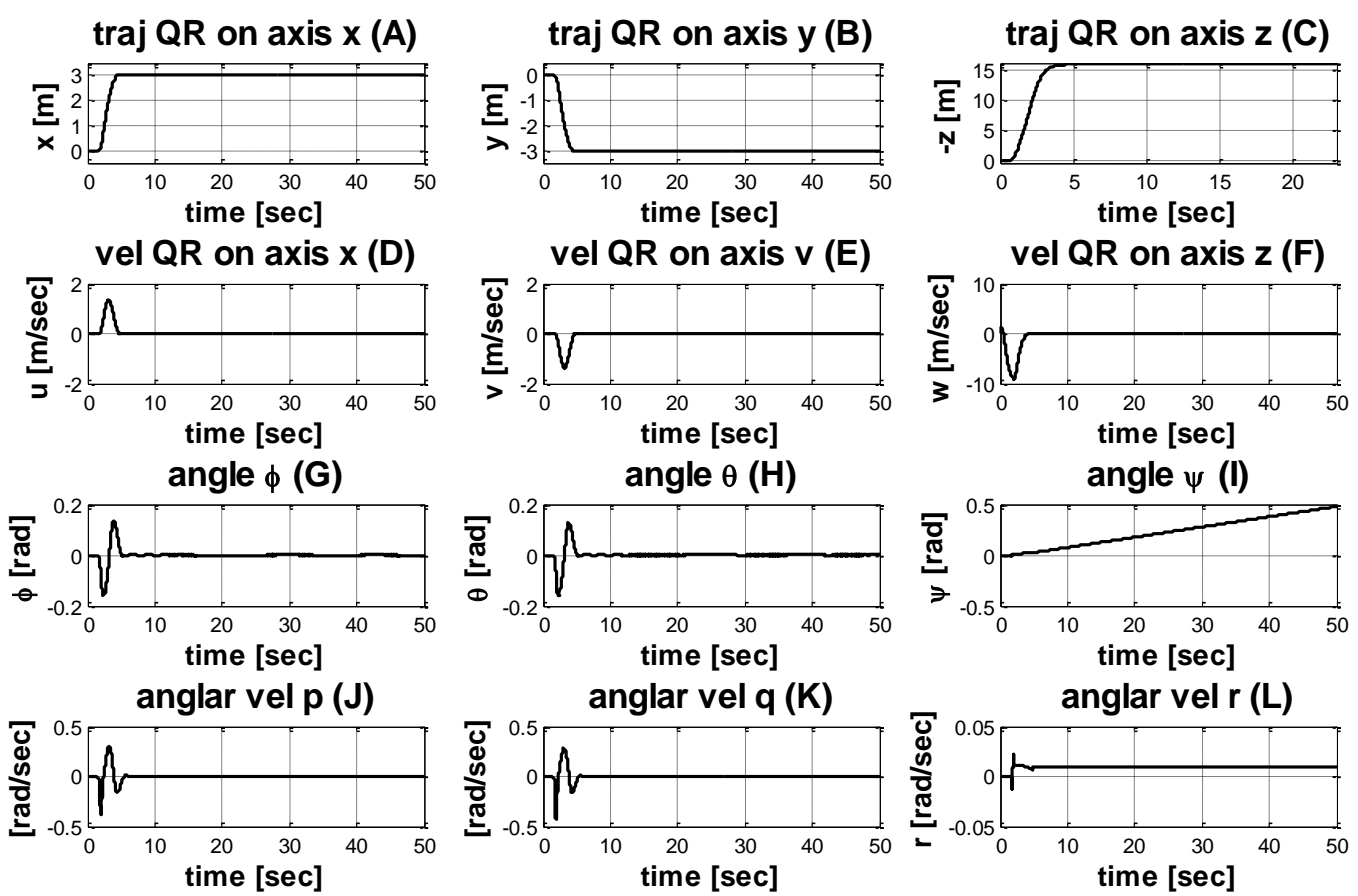

Figure (6) (A B C) linear positions $(x, y, z),($ D E F) linear velocities $(u, v, w)$, ( G H I) angler positions $(\phi, \theta, \psi)$ and (J K L ) angler velocities ( p, q, r) 

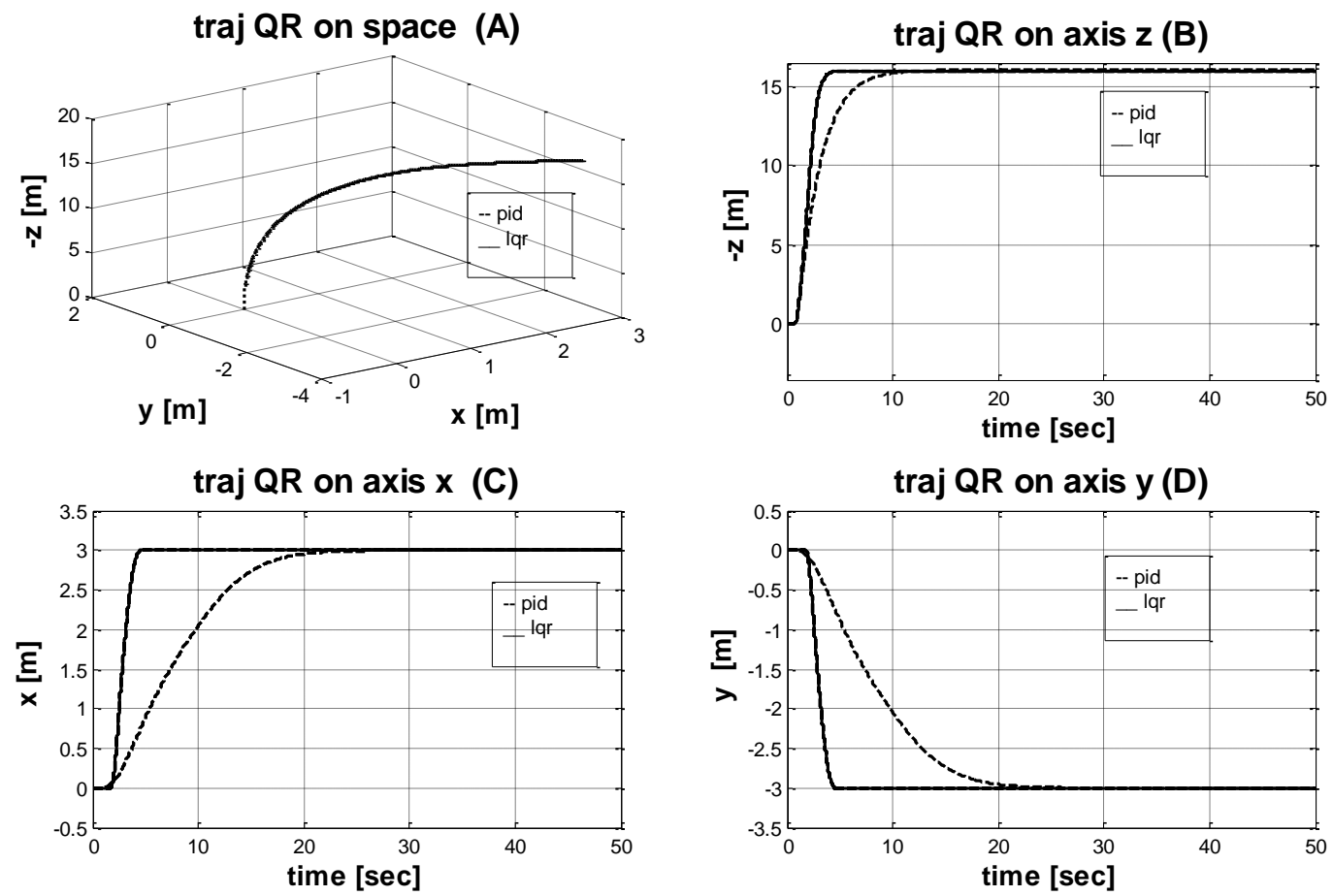

Figure (7) (A) 3 dimension trajectory quadrotor, B linear positions( z), (C) linear positions (x), (D) linear positions (y)

\subsection{Desired Trajectory}

Embedded Matlab function is carried out to create desired trajectory as shown in figures (89).

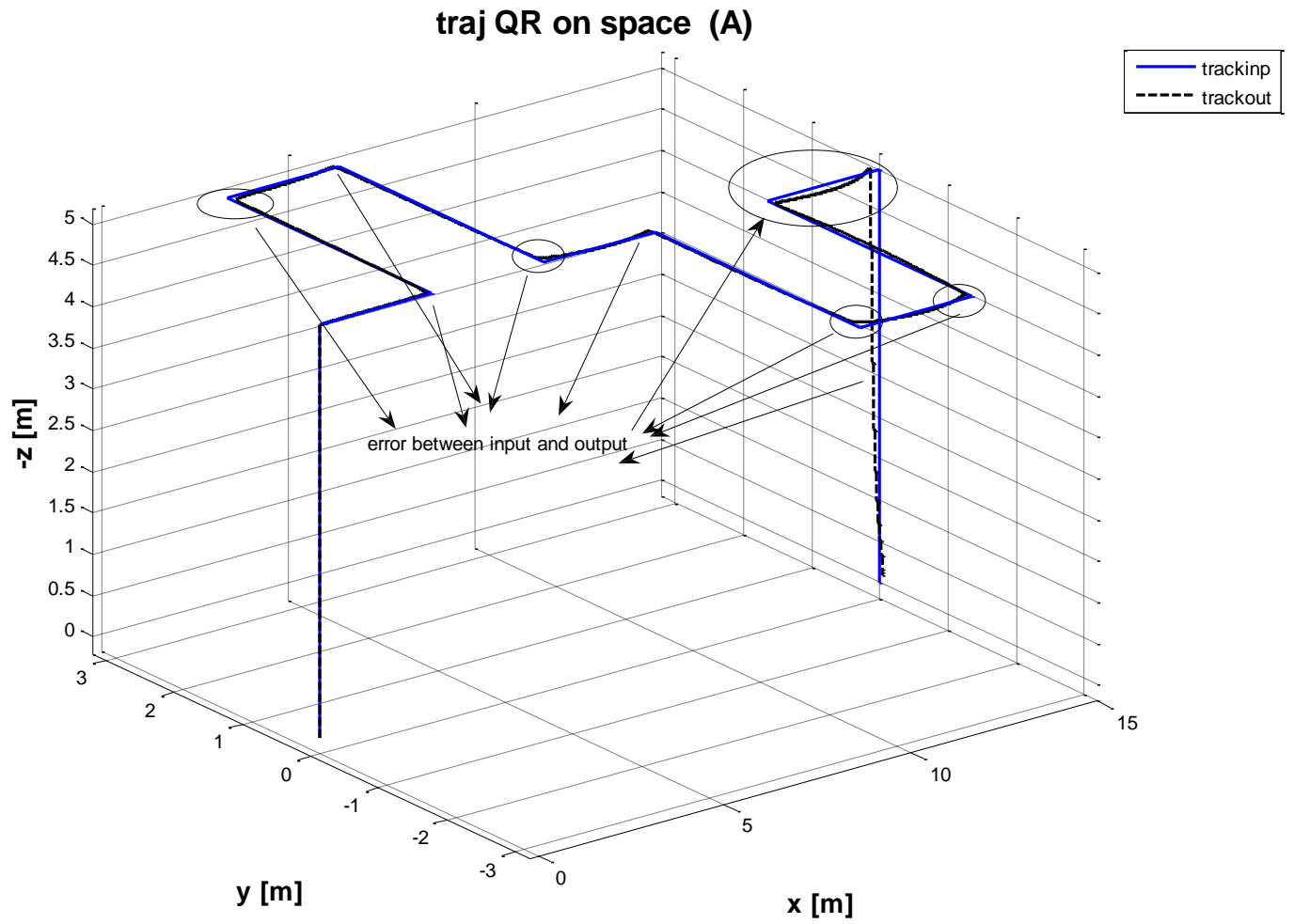

Figure (8) 3 dimension input trajectory and trajectory quadrotor, 


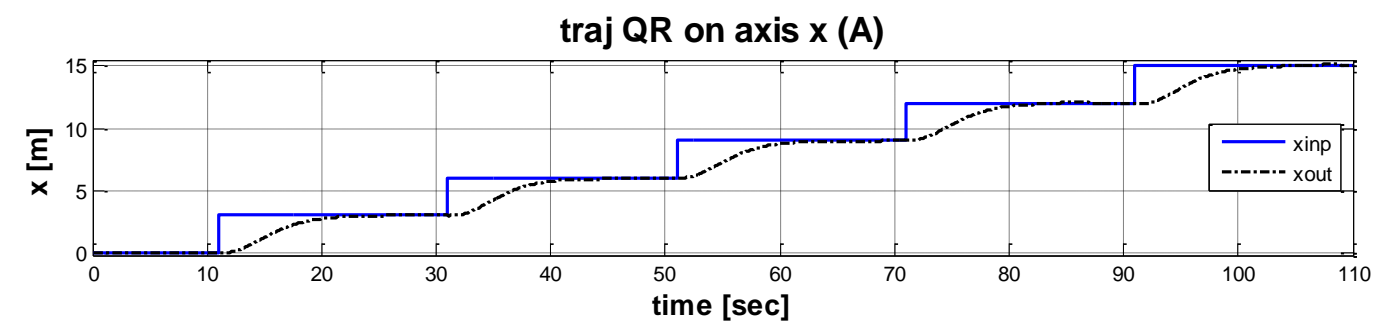

traj QR on axis y (B)

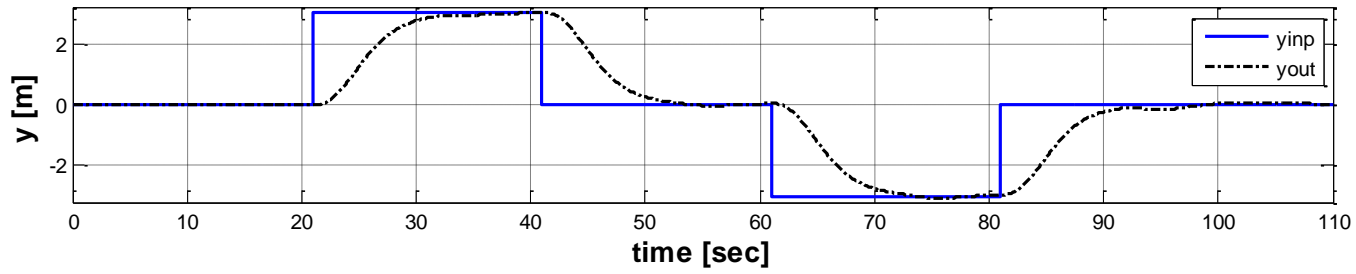

traj QR on axis x (C)

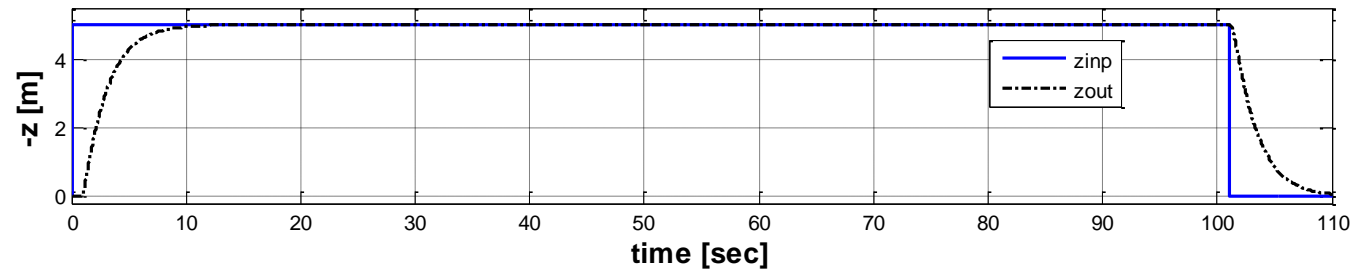

Figure (9) input trajectory and trajectory quadrotor in ( x), (A), (y) (B), (z),(C)

\section{Conclusion}

In this paper, a full non linear model starting from voltages input able to determine its own attitude through an onboard sensor modeling is carried out to have a desired trajectory. Ongoing research results in the development and implementation of an autonomous flight control system for a quadrotor helicopter are highlighted. Controlling a Vertical Taking-Off and Landing (VTOL) flying vehicle is basically dealing with highly unstable dynamics and strong axes coupling. The simulation results showed a satisfactorily performance of the proposed controllers adopted on the quadrotor in face off the presence of coupling and nonlinearties. Vertical flight, hover, landing and horizontal flight are among the considered flight features in a predetermined trajectory.

\section{References}

[1] S. Bouabdallah, P. Murrieri, and R. Siegwart. "Design and Control of an Indoor Micro Quadrotor. In Robotics and Automation", 2004. Proceedings. ICRA'04. 2004.

[2] J. Domigues, "Quadrotor Prototype", Master Thesis university Lisboa October 2009.

[3] P. Castillo, R. Lozano, and A. Dzul, Modelling and Control of Mini-Flying Machines (Springer-Verlag Series in Advances in Industrial Control).New York: Springer-Verlag, 2005.

[4] V. M. Martinez, "Modeling of the Flight Dynamics of a Quadrotor Helicopter". Masters Thesis Cranfield University2008.

[5] S.Robboz,Y.Ehawagy,OE.Abdelhamid, "Flight Dynamics Simulation of Quadrotor", The Fifth International Conference on Intelligent Computing and Information Systems http://icicis.edu.eg/ Ain Shams University June 30, 2011 Chairs.

[6] B,Nourghassemi, Development of the Control Algorithms for Autonomous Landing of Unmanned Aerial Vehicles, Master Thesis 2009. 
[7] C balls. Modelling and Linear Control of a Quadrotor, Masters Thesis Cranfield University, 2009.

[8] Pounds, P., Mahony, R., Hynes, P. and Roberts, J. (2002). Design of a Four- Rotor Aerial Robot. In: Proc. Australasian Conference on Robotics and Automation, Auckland, 27-29 November 2002.

[9] Benallegue, A., Mokhtari, A. and Fridman, L. (2006), "Feedback Linearization and High Order Sliding Mode Observer for a Quadrotor UAV", Proceedings of the 2006 International Workshop on Variable Structure Systems, June 2006,Alghero, Italy, pp. 365.

[10] Bouabdallah, S., Murrieri, P. and Siegwart, R. (2005), "Towards Autonomous Indoor Micro VTOL", Autonomous Robots, [Online], vol. 18, no. March, 2005.

[11] Bouabdallah, S., Noth, A. and Siegwart, R. (2004), PID vs LQ Control Techniques Applied to an Indoor Micro Quadrotor, Swiss Federal Institute of Technology. 